\title{
THE NUMBER OF POLYHEDRAL (3-CONNECTED PLANAR) GRAPHS
}

\author{
A. J. W. DUIJVESTIJN
}

\begin{abstract}
Data is presented on the number of 3-connected planar graphs, isomorphic to the graphs of convex polyhedra, with up to 26 edges. Results have been checked with the the number of rooted c-nets of R.C. Mullin and P.J. Schellenberg and Liu Yanpei.
\end{abstract}

\section{INTRODUCTION}

The set of 3-connected planar graphs is isomorphic with the set of polyhedral graphs. Tables of low-order polyhedral graphs were first given by Steiner in 1828, for references see [8]. Polyhedral graphs play an important role in the calculation of solutions of the squared rectangle and squared square problem as shown by Brooks, Smith, Stone, and Tutte [1]. They use the term c-nets for polyhedral graphs. In 1981 Duijvestijn and Federico published tables of polyhedral graphs up to order 22 and parts of order 23 and 24 [8].

A c-net is a three-connected planar graph. The order of a c-net is its number of edges. The dual of a c-net is also a c-net. The c-nets are constructed using Tutte's theorem, known since 1947 and published in 1961 [12].

Let $C_{n}$ be the set of c-nets of order $n$. If $s \in C_{n}$ is not a wheel, then at least one of the nets $s$ and its dual $s^{\prime}$ can be constructed from $\sigma \in C_{n-1}$ by addition of an edge joining two vertices. A wheel is a c-net with an even number of edges $E$, with one edge of degree $E / 2$ and $E / 2$ vertices of degree 3 . The degree of a vertex is the number of edges joining the vertex. Generation of c-nets of order $n+1$ out of order $n$ gives rise to many duplicate c-nets. These can be removed using an identification method described in 1962 [3, 4] and improved in 1978 [5]. The results presented here go as far as order 26 . The number tabulated in [8] contains a printing error in the order 22 and is corrected in the new tables. Independently, Dillencourt calculated 3-connected planar graphs up to order 26. He reminded me that a discrepancy occurred in the printed data in the tables of order 22. At that time we could compare results up to order 25. Both our results were the same. Recently, we could also compare results on order 26 . The number of c-nets of order 26 found by me was the same as the number found by Dillencourt ${ }^{1}[10]$.

Received by the editor February 16, 1995.

1991 Mathematics Subject Classification. Primary 05C30; Secondary 52B05.

Key words and phrases. Graph theory, 3-connected graphs, polyhedral graphs.

${ }^{1}$ Department of Computer Science, University of California, Irvine, California 92717-3425, email: dillenco@ics.uci.edu. 


\section{ROOTED C-NETS}

The concept of edge rooted graph was introduced by Tutte [13]. One edge is specified as the root and is directed by an arrow, and the two sides distinguished by labels $\mathrm{l}$ (left) and $\mathrm{r}$ (right). Since the arrow can be directed in two ways and in each case the sides can be labelled in two ways, four rooted graphs are produced from each edge, and the total number from a given graph is four times the number of edges. If the graph is symmetric, i.e., has a nontrivial automorphism, some of these will be isomorphic; the total number of distinct rooted graphs is $4 E / h$ where $E$ is the number of edges and $h$ is the order of the automorphism group of the graph. The number of rooted c-nets can be calculated (without constructing them) by Tutte's formula, and column 3 of Tutte's table gives these figures (R) up to 25 edges as given by Tutte [13]. Table 1 in Liu Yanpei [9] gives rooted c-nets up to order 36. Mullin and Schellenberg [11] have derived an explicit formula for calculating the rooted c-nets in each Euler class. That paper includes a table giving these numbers for all classes up to order 16. Additional data have been calculated using a formula for $q_{m, n}^{*}$ on page 216 with the help of the language "maple". The rooted c-nets can be calculated as soon as the order of the automorphism group is known. Table 1 is given in $\S 4$.

\section{Computer aspects}

The motivation for the calculation of c-nets of such high orders was the calculation of squared squares and $2 \times 1$ squared rectangles $[6,7,2]$. The generation of c-nets of orders 22, 23 and 24 was done on Sun Sparc workstations in the period September 5, 1990 to December 1990. The generation of c-nets of order 25 was carried out during the Christmas vacation week 1991, using four Sun Sparc workstations of the Faculty of Computing Sciences of the University Twente connected to the university network. The generation and identification of c-nets of order 26 was completed April 1992 on four HP workstations of the Faculty of Computing Sciences of the University Twente. The speed of the machines is 75 Mflops. It took more than $600 \mathrm{MB}$ of disk space in compressed form to store the c-nets.

\section{Results}

Results are tabulated by number of edges and the number of vertices. The number of c-nets as well as the number of rooted c-nets are given in Table 1, where NS means not selfdual and S means selfdual, V stands for number of vertices. Auto means order of the automorphism group. Since the dual of a c-net is a c-net, all c-nets can be obtained from Table 1 . The result has been verified by means of the formula on the number of rooted c-nets by Mullin and Schellenberg and Liu Yanpei. 
TABLE 1. Number of c-nets and rooted c-nets of orders 22 to 26

\begin{tabular}{|c|c|c|c|c|c|c|c|c|c|}
\hline \multicolumn{3}{|l|}{ Order 22} & \multirow{2}{*}{$\begin{array}{c}\text { C-nets } \\
4052\end{array}$} & \multirow{2}{*}{$\begin{array}{r}\text { Rooted C-nets } \\
356576\end{array}$} & \multicolumn{3}{|c|}{ Order 24} & \multirow{2}{*}{$\begin{array}{r}\text { C-nets } \\
137\end{array}$} & \multirow{2}{*}{$\begin{array}{r}\text { Rooted C-nets } \\
13152\end{array}$} \\
\hline $\mathrm{V}=10$ & NS & auto $=1$ & & & $\mathrm{~V}=10$ & & auto $=1$ & & \\
\hline $\mathrm{V}=10$ & NS & auto $=2$ & 365 & 16060 & & & auto $=2$ & 69 & 3312 \\
\hline $\mathrm{V}=10$ & NS & auto $=4$ & 24 & 528 & & & auto $=3$ & 1 & 32 \\
\hline \multirow{3}{*}{$\mathrm{V}=10$} & NS & auto $=8$ & 1 & 11 & & & auto $=4$ & 13 & 312 \\
\hline & & & $\overline{442}$ & 373175 & & & $\begin{array}{l}\text { auto }=6 \\
\text { auto }=8\end{array}$ & $\begin{array}{l}6 \\
4\end{array}$ & $\begin{array}{l}96 \\
48\end{array}$ \\
\hline & & & $4+42$ & S10175 & & & auto $=16$ & 1 & 6 \\
\hline $\mathrm{V}=11$ & NS & auto $=1$ & 102524 & 9022112 & & & auto $=24$ & 1 & 4 \\
\hline $\mathrm{V}=11$ & NS & auto $=2$ & 1663 & 73172 & & & auto $=32$ & 1 & 3 \\
\hline \multirow[t]{2}{*}{$\mathrm{V}=11$} & NS & auto $=4$ & 26 & 572 & & & & 233 & 16965 \\
\hline & & & 104213 & 9095856 & & & & & \\
\hline $\mathrm{V}=12$ & NS & auto $=1$ & 131718 & 11591184 & $\mathrm{~V}=11$ & & auto $=1$ & 78169 & 7504224 \\
\hline $\mathrm{V}=12$ & NS & auto $=2$ & 1486 & 65384 & & & auto $=2$ & 1559 & 74832 \\
\hline $\mathrm{V}=12$ & NS & auto $=4$ & 36 & 792 & & & auto $=3$ & 5 & 160 \\
\hline \multirow[t]{2}{*}{$\mathrm{V}=12$} & NS & auto $=8$ & 3 & 33 & & & auto $=4$ & 25 & 600 \\
\hline & & & $\overline{1332 \overline{43}}$ & 11657393 & & & $\begin{array}{l}\text { auto }=6 \\
\text { auto }=12\end{array}$ & $\begin{array}{r}13 \\
2\end{array}$ & 208 \\
\hline $\mathrm{V}=12$ & $\mathrm{~s}$ & auto $=1$ & 1817 & 150896 & & & & 79773 & 7580040 \\
\hline $\mathrm{V}=12$ & $\mathrm{~s}$ & auto $=2$ & 80 & 3520 & & & & & \\
\hline $\mathrm{V}=12$ & $\mathrm{~s}$ & auto $=4$ & 10 & 220 & & & & & \\
\hline \multirow{4}{*}{$\mathrm{V}=12$} & $\mathrm{~S}$ & auto $=22$ & 1 & 4 & $\mathrm{~V}=12$ & & auto $=1$ & 1255238 & 120502848 \\
\hline & & & 1908 & 163640 & & & auto $=2$ & 7631 & $\begin{array}{r}366288 \\
488\end{array}$ \\
\hline & & & & & & & auto $=4$ & 123 & 2952 \\
\hline & & & & & & & auto $=6$ & 12 & 192 \\
\hline \multirow{2}{*}{\multicolumn{3}{|c|}{ Order 23}} & & & & & $\begin{array}{l}\text { auto }=8 \\
\text { auto }=12\end{array}$ & $\begin{array}{l}5 \\
7\end{array}$ & $\begin{array}{l}60 \\
56\end{array}$ \\
\hline & & & C-nets & Rooted C-nets & & & auto $=24$ & 1 & 4 \\
\hline & & & & & & & auto $=48$ & 1 & 2 \\
\hline $\mathrm{V}=10$ & & auto $=1$ & 1235 & 113620 & & & & & \\
\hline $\mathrm{V}=10$ & & auto $=2$ & 156 & 7176 & & & & 1263032 & 120872850 \\
\hline \multirow[t]{3}{*}{$\mathrm{V}=10$} & & auto $=4$ & 13 & 299 & & & & & \\
\hline & & & 1404 & 121095 & $\mathrm{~V}=13$ & NS & auto $=1$ & 1460152 & 140174592 \\
\hline & & & & & & NS & auto $=2$ & 5199 & 249552 \\
\hline $\mathrm{V}=11$ & & auto $=1$ & 110015 & 10121380 & & NS & auto $=3$ & 15 & 480 \\
\hline $\mathrm{V}=11$ & & auto $=2$ & 2023 & 93058 & & NS & auto $=4$ & 27 & 648 \\
\hline \multirow[t]{3}{*}{$\mathrm{V}=11$} & & auto $=4$ & 44 & 1012 & & NS & auto $=6$ & 18 & 288 \\
\hline & & & $\overline{112082}$ & $102 \overline{15450}$ & & $\mathrm{NS}$ & auto $=8$ & 3 & 36 \\
\hline & & & & & & & & 1465414 & 140425596 \\
\hline $\mathrm{V}=12$ & & auto $=1$ & 704267 & 64792564 & & & & & \\
\hline & & auto $=2$ & 4977 & 228942 & & & & & \\
\hline \multirow[t]{8}{*}{$\mathrm{V}=12$} & & auto $=4$ & 58 & 1334 & $\mathrm{~V}=13$ & $\mathrm{~s}$ & auto $=1$ & 6490 & 623040 \\
\hline & & & $\overline{709302}$ & 65022840 & & $\mathrm{~S}$ & auto $=2$ & 144 & 6912 \\
\hline & & & & & & $\begin{array}{l}S \\
\text { S }\end{array}$ & $\begin{array}{l}\text { auto }=3 \\
\text { auto }=4\end{array}$ & $\begin{array}{r}12 \\
7\end{array}$ & $\begin{array}{l}384 \\
168\end{array}$ \\
\hline & & & & & & $\mathrm{S}$ & auto $=6$ & 8 & 128 \\
\hline & & & & & & $\mathrm{S}$ & auto $=8$ & 3 & 36 \\
\hline & & & & & & $\mathrm{S}$ & auto $=12$ & 2 & 16 \\
\hline & & & & & & $\mathrm{s}$ & auto $=24$ & 1 & 4 \\
\hline & & & & & & & & 6667 & 630688 \\
\hline
\end{tabular}


TABLE 1. (continued)

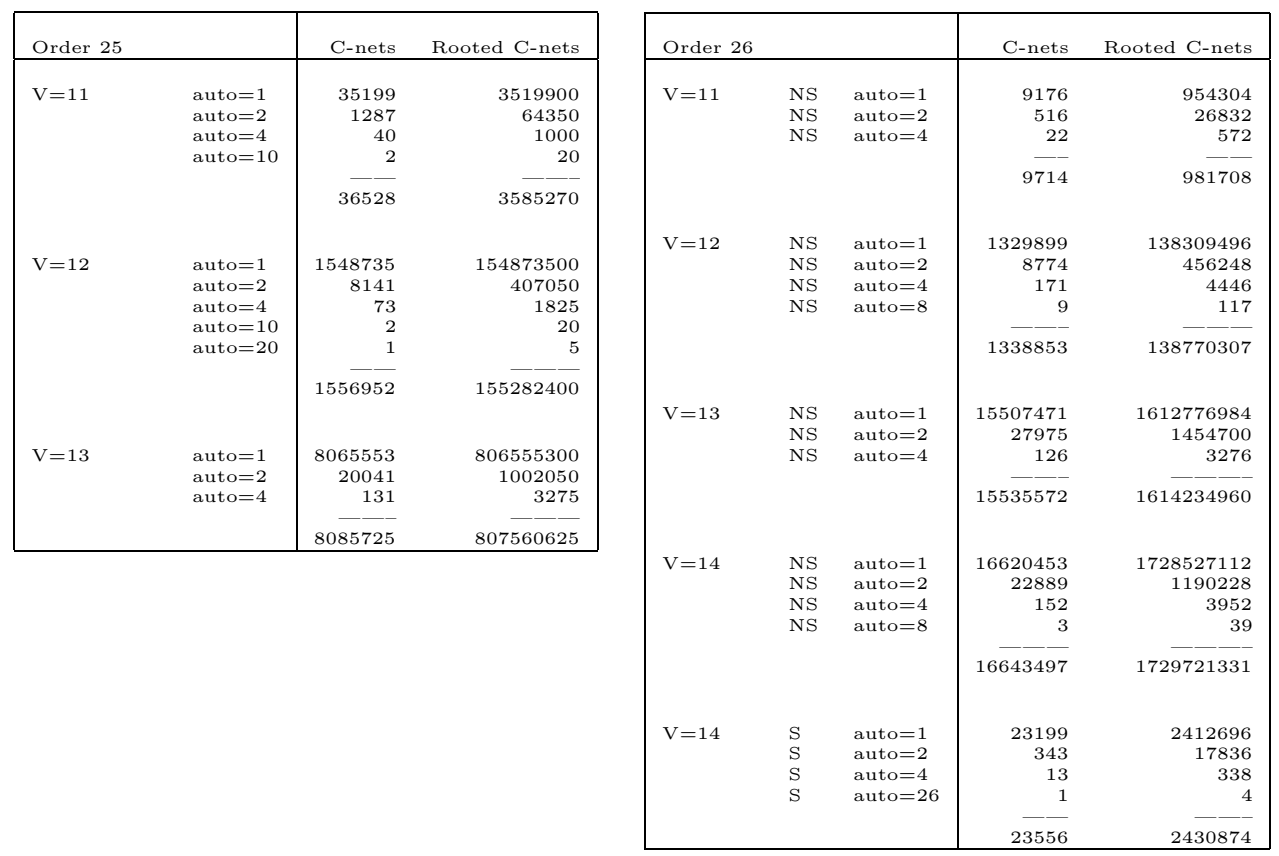

\section{Acknowledgement}

The constant support of Bert Helthuis of the laboratory of the Faculty of Computer Sciences in using the computer systems is gratefully acknowledged.

\section{REFERENCES}

1. R.L. Brooks, C.A.B. Smith, A.H. Stone, and W.T. Tutte, The dissection of rectangles into squares, Duke Mathematical Journal 7 (1940), 312-340. MR 2:153d

2. A.J.W. Duijvestijn, Simple perfect squared squares and $2 \times 1$ squared rectangles of order 26 , Mathematics of Computation,65(1996)to appear.

3. __ Electronic computation of squared rectangles, Ph.D. thesis, Technological University Eindhoven, 1962. MR 26:2036

4. _ Electronic computation of squared rectangles, Philips Research Reports 17 (1962), 523-612.

5. - Algorithmic calculation of the order of the automorphism group of a graph, Memorandum 221, Twente University of Technology, The Netherlands, 1978.

6. _ Simple perfect squared squares and $2 \times 1$ squared rectangles of orders 21 to 24, Journal of Combinatorial Theory B 59 (1993), 26-34. MR 94c:05017

7. Simple perfect squared squares and $2 \times 1$ squared rectangles of order 25, Mathematics of Computation 62 (1994), 325-332. MR 94c:05023

8. A.J.W. Duijvestijn and P.J. Federico, The number of polyhedral (3-connected planar) graphs, Mathematics of Computation 37 (1981), 523-532. CMP 95:17

9. Yanpei Liu, On the number of rooted c-nets, Journal of Combinatorial Theory B 36 (1984), 118-123. MR 85k:05060

10. Dillencourt M.B., 1991, private communication.

11. R.C. Mullin and P.J. Schellenberg, The enumeration of c-nets via quadrangulations, Journal of Combinatorial Theory B 4 (1968), 259-276. MR 36:1362 
12. W.T. Tutte, A theory of 3-connected graphs, Kon. Nederl. Akad. Wetensch., vol.64, 1961, (= Indag. Math. 23 (1961), 441-455). MR 25:3517

13. W.T Tutte, A census of planar maps, Canadian Journal of Mathematics 15 (1963), 249-271. MR 26: 4343

Department of Informatics, Technological University Twente, Enschede, The NETHERLANDS

E-mail address: infdvstn@cs.utwente.nl 